\section{Role of compression after liposuction}

\author{
Håkan Brorson, Barbro Svensson, Karin \\ Ohlin \\ Department of Plastic and Reconstructive \\ Surgery, Malmö University Hospital, \\ Sweden
}

\section{Introduction}

Liposuction, which is a very effective treatment modality to reduce lymphedema, can be performed as soon as the pitting component had been corrected by a phase of initial compression (Figure 1). Just like after conservative treatment, the use of compression garments after liposuction is very important to maintain the outcome.

Garments should be prescribed in such numbers so that the edema does not recur. A common mistake is that the patient receive one, or if lucky two, garments, after treatment. When the arm or leg is swollen again the patient comes back to the therapist and treatment starts again, and so on. Instead the patient must be followed up at his needs in order to prevent recurrence. This means that treatment must be individualized. An elderly woman may need two garments every 6 months, while a young active patient with a heavy work may need two garments every month. One can draw a parallel to the dosage of insulin to a patient with diabetes that in the same way must be individualized. Nobody prescribes two vials of insulin for 6 months, when the patient needs that same amount for one month.

The purpose of compression therapy is to increase the interstitial pressure so that the capillary filtration is decreased. When treated with standard compression garments a study showed a reduction of the excess volume (1680 mL; range 670-3320) after two weeks with 20\% (range 5-37) corresponding to $338 \mathrm{ml}$ (range 95-1225). ${ }^{1}$ Studies of treatment for 6 months have shown a reduction of excess volume of $17 \%$ (range 16-52), corresponding to a volume of $139 \mathrm{~mL}$ (range 150-345). ${ }^{2}$

Compression garments can be used at the onset of symptoms to possibly prevent the development of lymphedema. Garments that are used throughout the day (15 years' followup $)^{3}$ as well as only daytime (6 months followup $)^{2}$ prevent the edema to recur.

Compression garments must be ordered by a qualified and experienced lymphedema team, consisting of a lymph therapist with a basic education in physiotherapy or occupational therapy and a physician. The team should have vast knowledge regarding various compression trademarks and how to take measurements for ordering of garments. In order to increase compliance and feedback, the team should never let the patient have the measurements for ordering of garments taken by a retailer outside of the team.

\section{Ordering of garments}

When ordering for compression garments the following must be considered: i) compression class; ii) material; iii) size; iv) design; v) the patient's ability to take on/off the garment and the ability to care for it.

\section{Compression class}

The compression classes (CCL) provisionally adopted by the European Standardization Committee are summarized in Table $1 .^{4}$

Compression garments for the arm and hand are usually ordered in CCL 2 . In case of incipient symptoms CCL 1 can be sufficient. Garments for the leg often require CCL 3 or more. If the lymphedema requires higher compression, a combination with an additional garment on top on the first one can be used. This increases the compression further and can be easier to put on than just one garment in very high compression class. In severe cases another leg long garment may be needed, either a round knitter or a flat knitted.

\section{Materials and Methods}

Materials and production methods vary between different manufacturers. There are circular knitted garments without a seam and flat knitted with a seam. The higher compression classes are usually flat knitted. It should be noted that each class is within a compression interval, and within the compression class different materials may have higher or lower compression. Verify how long the manufacturer guarantees that the garment lasts for the specified compression. Garments in softer materials have a shorter durability and must be replaced more often, even though the compression is the same as that of a more rigid garment.

Patients with sensitive skin may need to try different brands. If the patient encounters irritation at the elbow or at the back of the knee a soft lining can be sewn into the sock. There are also modifications in the knitting technique that decreases skin irritation at these locations. There are also different brands of silicone or hydrogel plates, to be put under the garment that will relieve irritated skin.

\section{Size}

Compression stockings are produced in different standard sizes or made to measure:
Correspondence: Håkan Brorson, Department of Plastic and Reconstructive Surgery, Malmö University Hospital, SE-205 02 Malmö, Sweden. E-mail: hakan.brorson@med.lu.se

This work is licensed under a Creative Commons Attribution 4.0 License (by-nc 4.0).

(C) Copyright H. Brorson et al., 2016

Licensee PAGEPress, Italy

Veins and Lymphatics 2016; 5:5990

doi:10.4081/vl.2016.5990

- Standard size garments: i) advantage: the patient receives the garment at once; measuring is simpler; ii) disadvantage: do not have enough widths and lengths to suit all; smaller selection of materials; smaller selection of models.

- Made to measure garments: i) advantages: optimal fit; greater possibility when re-measuring in order to gradually reduce the size of the garment as the edema is reduced; ii) disadvantages: more extensive measuring; delivery time; the cost.

If made to measure is selected after treatment, the patient must either use a standard garment or bandage the extremity until the custom made garment has been delivered. Later on, when measuring for the next garment, the measurements must be compared with the previous order to check that the garment gives the intended effect. The technique when measuring varies from brand to brand. See the instructions of each manufacturer.

\section{Design}

Compression stockings are available in various models. You can also choose various fasteners, such as silicone ribbon at the top of the garment or a band around the waist. Each manufacturer provides information about the different varieties available. Which one you choose depends on: i) edema location; ii) the patient's body constitution; iii) the patient's wishes; iv) how compression stocking works in daily activities.

Taking on and off the garment and maintenance of the garment.

Check that the patient can maintain and manage to take on and off the garment. If the patient has problems one must arrange for someone to help out, such as a relative or home care/district nurse, who also may need instructions. At follow-up, check that this has worked. The manufacturers provide different types of aids that facilitate the procedure.

\section{Use}

The various alternatives depend on the severity of the edema: i) continuously throughout the day; ii) continuously during daytime 
(the garment must be taken on in the morning); iii) only use it when performing strenuous work (prolonged static or heavy work, where patients experience that the extremity gets swollen) and at long journeys by plane or car. After a few weeks, it is important to verify that compression stockings have the intended effect. Rash after use may be due to an allergic reaction to detergents, or to the garment material, which is very uncommon. If the excess volume has increased at follow-up, this can be due to several factors: i) bad patient compliance; check the patient's motivation and every day routines; ii) the patient has not understood how the garment must be taken care of or has taken it on in the wrong way (for example not pulled it high enough); iii) incorrect measurements have been taken - check them; iv) insufficient compression class - increase compression class at the next order; v) progress of underlying malignancy.

\section{Costs}

The life span of a compression garment varies between 3 and 6 months, provided that the patient has two garments to switch between. Two garments are required so that the patient every day can take on a newly washed garment. At the first time only one garment is ordered to evaluate the fit and to be able to make any corrections. Then the second garment is promptly ordered. Henceforth two garments should be ordered at each occasion. The minimum amount of compression garments for an arm lymphedema is two every 6 months. If gloves/gauntlets are needed, 2-3 garments every 6 months may be needed since they wear out faster. For legs new garments may need to be reordered every 3-6 months depending on activity level. Very active patients and children may need up to 6 stockings every six months. There should be no upper limit to how many garments that need to be ordered. Additional garments may be needed at the start of treatment.

Funds must be set aside anyway so that patients can obtain sufficient numbers of compression garments as described above.

\section{Controlled compression therapy - gradually increasing compression}

When an untreated edema is subjected to compression, the excess volume is reduced and compression must be adapted continuously. This can be done with bandaging or controlled compression therapy. The methods can be used individually or in combination. It is very important that treatment outcome is checked regularly with excess volume measurements and that treatment continues until there is minimal or no pitting (Figure 1). No manual lymph drainage is needed so the treatment is less time consuming and the patient does not need to be on sick leave. Also manual lymph drainage lacks evidence in reduction of the excess volume. ${ }^{5-7}$

\section{How can compression be increased?}

There are several ways to increase compression pressure: i) decrease circumferential measurements of garments; ii) increase compression class; iii) multilayer; iv) amount of garments; v) take in existing garments.

As long as the extremity shows pit on pressure the garment can be reduced in size by using a sewing machine. The circumference can be decreased at $0.5-1 \mathrm{~cm}$ increments at regular controls and can also be easily made by the patient when necessary. After 3-4 times the patient needs new garments in a smaller size. Every time new garments are ordered they are ordered in a smaller size than last time, if ordering made to measure garments. The method is an alternative to bandaging in connection with the initial edema reduction. ${ }^{1}$

Controlled compression therapy can be used as the only treatment. It can also be used while waiting for other physical-medical methods or surgical treatment as may be indicated.

\section{Treatment and Results}

For arm lymphedema CCL 2 is mostly used. Occasionally CCL 3 may be necessary. The glove can be made of the same material or thinner material (glove for burns) if the patient's hand edema is not as pronounced. At follow-up, measurements for garments are taken much tighter than the recommendations specified by the manufacturer in order to increase compression and to get sufficient compression for a longer time. For leg lymphedema CCL 3 (leg long) and CCL 2 (knee long) are used. This particular measuring requires an experienced therapist.

Further follow-up and reduction of the compression garments is made when needed and at scheduled visits at 1 and 3 months. Up to the

Table 1. Compression classes as per the European Standardization Committee.

\begin{tabular}{lccl} 
Class No. & Description & Value & \\
CCL I: & Mild & $15-21$ & $\mathrm{~mm} \mathrm{Hg}^{*}$ \\
CCL II: & Moderate & $23-32$ & $\mathrm{~mm} \mathrm{Hg}$ \\
\hline CCL III: & Strong & $34-46$ & $\mathrm{~mm} \mathrm{Hg}$ \\
CCL IV: & Very strong & $49-$ & $\mathrm{mm} \mathrm{Hg}$
\end{tabular}

CCL, compression class. *Compression at the ankle. The values indicate the compression exerted by the compression garments at an hypothetical cylindrical ankle.

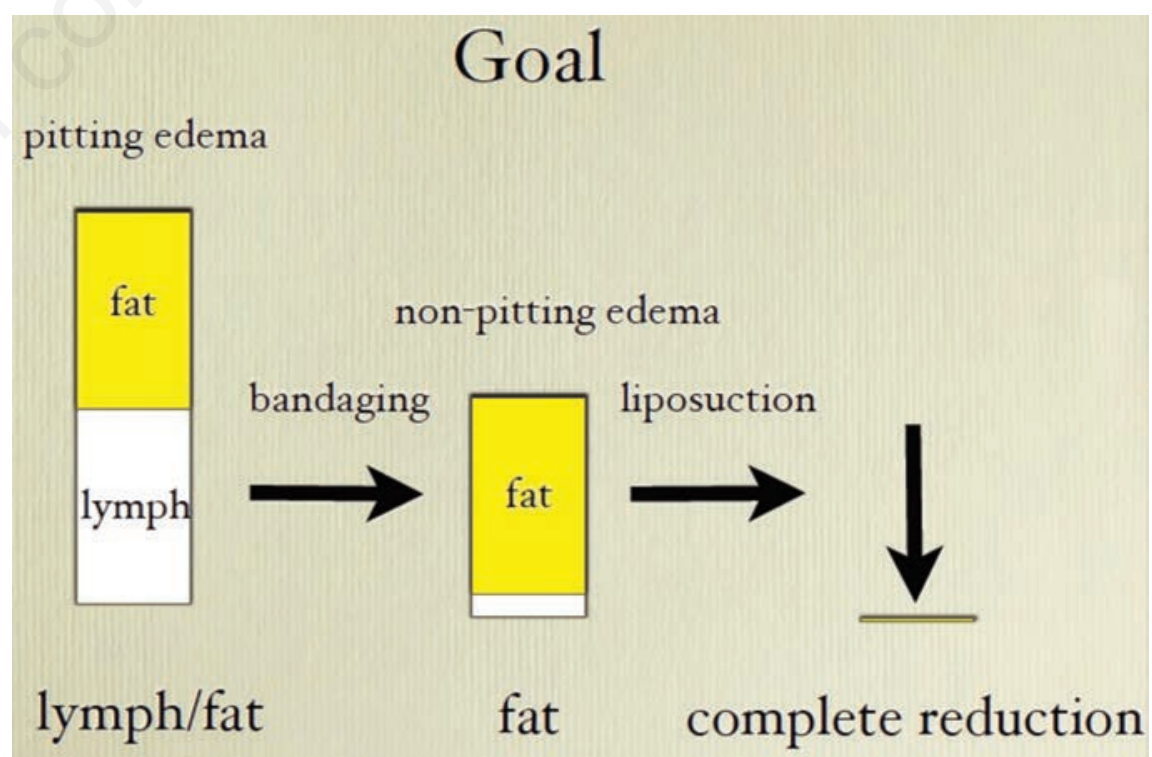

Figure 1. Transforming a pitting lymphedema to a non-pitting one. If the remaining excess volume is still a problem for the patient liposuction can be performed to get complete reduction. 
3 -month control, when new garments are ordered in smaller size, the patient made 3-4 reductions (using a sewing machine), when he/she notices that the compression decreases. Further follow-up with ordering of new garments is done every 3 months until the maximum edema reduction is achieved, i.e., when there is no minimal pitting (3-4 mm for arms and 5-6 $\mathrm{mm}$ for legs). This is usually achieved after 6-12 months of treatment. The excess volume is measured at each control.

If the excess volume is stable at 1 year, the patient is provided with garments for the next 6 months. If still stable at 1.5 years the patients is seen at 2 years. Then, if stable, the patient needs only to be seen once a year and is provided with all the garments for the following year, and so on.

Controlled compression therapy with compression garments shows an excess volume reduction of $47 \%$ (range 2-80) after one year. Already after two weeks with a standard garment a reduction of $20 \%(340 \mathrm{~mL})$ is achieved. The results are permanent after 2 years. These results have been achieved with excess volumes of on average 1.7 liters (0.7-3.3 l), the relative volume of 1.6 (range 1.3 to 2.7 ) and long duration (mean duration 8 years, range 119)..$^{1,8}$ Lymphoscintigraphy shows signs of increased mobilization of lymph after 3 months of compression therapy. After 12 months there is no difference compared with values before treatment. ${ }^{9}$ This is consistent with findings from combined physiotherapy. ${ }^{10}$

\section{Follow-up (summary)}

Initial follow-up visits are made with a few weeks apart and can then, at steady state, be reduced to 1-2 times per year. Treatment is lifelong.

The control procedures must be included: i) measurement and calculation of absolute and relative excess volume; ii) review of the compression garments' fit and elasticity. When necessary it is reduced in size by the use of a sewing machine and/or new garments are ordered; iii) at steady state with persistent troublesome excess volume and minimal or no pitting liposuction can further reduce the excess volume.

\section{Conclusions}

All five strategies of increasing compression can be combined to treat leg lymphedema. Three of the strategies are useful also when you want to shape specific parts of the leg. You localize the parts needing extra compression by comparing circumferences and by estimat- ing pitting. Finally most important is to involve the patient in the decisions. It is the patient that has to do the job, putting on the garments every day and keep them on day and night. If they feel they have a choice in which way to go they will most likely do their part.

We used different combinations of increasing compression, the patients needed different amount of garments and different intervals between checkups, but the important thing is to help them never loose control over their lymphedema and help them back on track if the edema temporarily gets worse.

\section{Advice to the patient}

The patient should be advised about: i) taking on the garment; ii) seeing the manufacturers' instructions; iii) rubber gloves can be used for better grip; iv) Easy Slide (BSN Medical, Kent, WA, USA) is a simple pull-on tool for both leg and arm; v) anti-slip mat on the floor can make it easier to brace against and herd up the sock over the heel; vi) the material should be distributed evenly over the limb so that pleats are avoided. Pleats lead to folds and increased pressure; vii) it is important that the arm garment is pulled high enough so that the elbow marking is at the right level. The elbow and the terminations need to be at the correct level. Poorly fitted garment leads to lower pressure and risk of chafing.

\section{Care}

To maintain the garment's properties during 3-6 months it must be handled according to the manufacturer's instructions.

The garment is washed daily to restore its compression and shape and to remove the salt precipitations from perspiration. The salt dries the skin and increases the risk of skin irritation.

The garment should be left to dry horizontally to avoid stretch. It dries faster if you first roll it in a towel and gently squeeze the water.

Soaking, use of fabric softener and tumbling harm the compression garments.

Fat, oil based skin creams should not be used because they can dissolve the rubber thread in rubber-based garments. Use moisturizing lotions instead.

\section{Miscellaneous}

Skin rash after using the garment may be due to allergic reaction to detergent or the material used.

If a new garment feels too tight, it can be stretched over an object while drying after washing, for example a bottle that is somewhat wider the extremity for a few hours.

Taking in the garment can be made between scheduled visits if it is too loose or as part of the treatment for controlled compression therapy. The patient can be instructed to do it by him/herself.

Thick seams, for example on the fingers, may need to be stretched somewhat before use, to prevent chafing.

During hot summer days, it is wise to bathe or shower with compression garment on and let it dry on the skin. This provides a cooling effect.

\section{References}

1. Brorson H, Svensson H. Liposuction combined with controlled compression therapy reduces arm lymphedema more effectively than controlled compression therapy alone. Plast Reconstr Surg 1998;102:1058-67.

2. Swedborg I. Effects of treatment with an elastic sleeve and intermittent pneumatic compression in post-mastectomy patients with lymphoedema of the arm. Scand J Rehabil Med 1984;16:35-41.

3. Brorson H. From lymph to fat: liposuction as a treatment for complete reduction of lymphedema. Int $\mathrm{J}$ Low Extrem Wounds 2012;11:10-9.

4. European Committee for Standardisation (CEN/TC 205WG2). Medical compression hoisery. European Standard CEN/ENV 12718, 2001.

5. Andersen L, Hojris I, Erlandsen M, Andersen J. Treatment of breast-cancerrelated lymphedema with or without manual lymphatic drainage--a randomized study. Acta Oncol 2000;39:399-405.

6. Dayes IS, Whelan TJ, Julian JA, et al. Randomized trial of decongestive lymphatic therapy for the treatment of lymphedema in women with breast cancer. J Clin Oncol 2013;31:3758-63.

7. Javid SH, Anderson BO. Mounting evidence against complex decongestive therapy as a first-line treatment for early lymphedema. J Clin Oncol 2013;31:3737-8.

8. Brorson H, Svensson H. Complete reduction of lymphoedema of the arm by liposuction after breast cancer. Scand J Plast Reconstr Surg Hand Surg 1997;31:137-43.

9. Brorson H, Svensson H, Norrgren K, Thorsson 0. Liposuction reduces arm lymphedema without significantly altering the already impaired lymph transport. Lymphology 1998;31:156-72.

10. Ketterings C, Zeddeman S. Use of the Cscan in evaluation of peripheral lymphedema. Lymphology 1997;30:49-62. 